\title{
Effects of dry period length on follicular dynamics in early lactating Holstein cows
}

\author{
A Soleimani ${ }^{1,2}$, A Heravi Moussavi ${ }^{2}, \mathrm{M}$ Danesh Mesgaran ${ }^{2}, \mathrm{~S} \mathrm{Safa}^{2}$ \\ ${ }^{1}$ Islamic Azad University- Kashmar Branch, Kashmar, Khorasan Razavi, Islamic Republic of Iran, \\ ${ }^{2}$ Dept of Animal Science, Ferdowsi University of Mashhad, Mashhad, Khorasan Razavi, Islamic Republic of Iran \\ Email:bbheravi@yahoo.com
}

Introduction For profitable production of milk, a nonlactating or dry period is established between lactations of dairy cow. There has been substantial recent interest in shortening dry periods. Physiological studies show that the changes the udder goes through to prepare for the next lactation take about three weeks, and yet current advice is that the most appropriate dry period for a modern dairy cow is 45 to 60 days. More recent studies show good reason to question that advice, and suggest dry periods of 30 to 35 days have no detrimental effect on production, but information relating the effects of dry period to subsequent reproductive performance of lactating cows is sparse (Grummer, 2007). Also, the effect of this management change on follicular dynamic has not been adequately evaluated. Therefore, the objective of the present study was to determine the effects of dry period length on follicular dynamics in early lactating Holstein cows.

Materials and methods Holstein cows $(\mathrm{n}=24)$ were randomly assigned in 1 of 2 treatments: 1$)$ traditional $60 \mathrm{~d}$ dry period and 2) a shortened $35 \mathrm{~d}$ dry period. Holstein cows were blocked by parity ( $2^{\text {nd }}$ and $3^{\text {rd }}$ to $5^{\text {th }}$ ), their previous 305 -day milk yield and expected calving dates. All cows were fed by routine ration of farm (total mixed diet) twice a day at 0800 and $1400 \mathrm{~h}$ and had at all time free access to water. To monitor follicular parameters, ultrasound measurements of follicular activity were made on alternate days from day 10 to 35 postpartum to determine the characteristics and fate of the $1 \mathrm{st}$ follicular wave, using a 7.5 MHz rectal transducer (Medison SA 600V, Seoul, Korea). Dominant follicle development was characterized by follicular mapping of recorded ultrasound images (Heravi Moussavi et al., 2007). Follicular recruitment during the $1^{\text {st }}$ follicular wave after parturition was evaluated by quantification of the numbers of 5-10 mm follicles on day 10 and 14. A dominant follicle was defined as a follicle that was $>10 \mathrm{~mm}$ in diameter in the absence of other large (>9 mm) growing follicles. The data were analysed using the General Linear Model (GLM) procedure of SAS (2001) for a completely randomised design.

Results Dry period length had no effect on follicular parameters and days postpartum to first ovulation (Table 1). The number of medium-sized follicles (5 to $10 \mathrm{~mm}$ ) present on d 10 and 14, diameter of the largest follicle on $\mathrm{d} 10$ (at first ultrasound), diameter of the first dominant follicle on $\mathrm{d} 14$, maximum diameter of the first dominant follicle, number of days until detection of a follicle $\geq 10 \mathrm{~mm}$ in diameter, and days to first ovulatory follicle were all similar among the treatments. Our results are in contrast to Gumen et al. (2005) study that showed follicle diameter at first ultrasonography, days to detection of first 10-mm follicle, and days to first postpartum ovulation were impacted by dry period length.

Table 1 Ovarian follicles and development during the first follicular wave postpartum in cows with 60 and 35 d dry periods.

\begin{tabular}{lllll}
\hline & \multicolumn{3}{c}{ Dry period lengths, $\mathrm{d}$} & \\
\cline { 2 - 5 } Parameter & 60 & 35 & $\mathrm{SE}$ & $\mathrm{P}$ \\
\hline Number of follicles (5-10 mm in diameter) on day 10 & 4.25 & 3.83 & 0.570 & 0.61 \\
Number of follicles (5-10 mm in diameter) on day 14 & 5.00 & 5.10 & 0.631 & 0.92 \\
Diameter of the largest follicle at first ultrasound, mm & 10.66 & 10.54 & 0.843 & 0.91 \\
Diameter of first dominant follicle on d 14, mm & 13.37 & 12.95 & 0.844 & 0.73 \\
Diameter of the first dominant follicle, mm & 19.41 & 19.58 & 1.284 & 0.92 \\
Number of days until detection of a follicle $\geq 10 \mathrm{~mm}$ in diameter & 11.58 & 11.66 & 0.650 & 0.92 \\
Days postpartum to first ovulation, d & 27.66 & 27.08 & 2.776 & 0.88 \\
\hline \hline
\end{tabular}

Conclusions The results of this study demonstrated that the dry period lengths had no apparent effect on follicular dynamic.

Further studies are needed to evaluate the impact of shortening dry period on follicular dynamic and reproduction parameters.

Acknowledgements The authors gratefully acknowledge the managers of Moghoofat Malek dairy farm for providing the opportunity to carry out this experiment in the farm and also the staff for their assistance in this experiment and excellent care of the cows.

\section{References}

Grummer. R.R.2007. Strategies to improve fertility of high yielding dairy farms: Management of the dry period. Theriogenology. 68S. S281-S288

Gumen, A, R. R. Rastani, R. R. Grummer, and M. C. Wiltbank .2005. Reduced Dry Periods and Varying Prepartum Diets Alter Postpartum Ovulation and Reproductive Measures. J. Dairy Sci. 88, 2401-2411

Heravi Moussavi, A., R.O. Gilbert., T.R. Overton., D.E. Boauman., W.R. Butler (2007). Effect of feeding fish meal and n3 fatty acid on ovarian uterin responses in early lactating dairy cows. J.Dairy Sci. 90, 136-144 